\title{
Mutation Rate in Duchenne Muscular Dystrophy
}

\author{
H. K. GOSWAMI and B. D. CHAURASIA \\ From the Department of Botany, Government Science College and the Department of Anatomy, G.R. Medical College, \\ Gwalior, India
}

The estimation of mutation rates in our own species has emerged as an integral part of human genetic research. Prerequisites for existing methods of calculating mutation rates are the total birth statistics and/or frequency of patients in the whole population. Unfortunately, no such records are being maintained adequately in India; a fact which was painfully realized by Haldane (1965). It is for this reason, that no contribution from this country has been made to the estimation of mutation rates, while data have been accumulated for various diseases in most parts of the world (see Crow, 1961; Sutton, 1965).

The most common estimates have been for haemophilia and Duchenne muscular dystrophy. The last named disease was recorded during a survey during 1964 in the township of Agar. Recently (on the advice of Professor Sorsby), we tried to locate a few more cases of muscular dystrophy in the Central Province. In the present paper, family and population data are included and the mutation rate is calculated.

\section{Material}

The birth statistics have been available only for the Agar sample. Therefore, the mutation rate is calculated for this sample by the indirect method. The other two samples are taken from small villages and the indirect or semi-direct method of calculation (Crow, 1961) is not applicable.

\section{Calculation (Indirect Method)}

A sibship with two males with muscular dystrophy and one probably normal sib appeared suddenly in an out-breeding Vaishya family. No such anomaly is recorded among individuals of 4 preceding generations. Family data are shown in the Table and the pedigree is given in the Figure. Considering the hereditary nature of muscular dystrophy and that it is X linked (Snyder, 1941; Sutton, 1965) it must be assumed that the mother of the sibship (IV.5) was heterozygous for the recessive allele. She could

Received 18 September 1970. have either inherited this allele from a parent who carried it or her Hh genotype could have been the results of a mutation in one of the two gametes of her parents. The latter possibility seems more plausible since her two brothers are reported to be normal and her sister (IV.9) has given birth to two normal sons.

The propositus (V.3) in this family is the first case of muscular dystrophy to be medically recorded in Agar. There have been 11,840 births ( 6845 males) in Agar in the period under consideration (19531966). The approximate mutation rate can be calculated by the following indirect method of Penrose (1961).

$$
\mathbf{M}=\frac{1}{3} \times \mathbf{f}(1-\mathrm{F}),
$$

where $f$ is the incidence of patients under consideration among the males and $F$ is the relative fitness of the patients. Therefore

$$
M=\frac{1}{3} \times \frac{2}{6845} \times 1=\frac{2}{20,535}
$$

which is equal to 9.7 per 100,000 gametes or $9 \cdot 7 \times 10^{-5}$ gametes per generation.

This estimation is in agreement with the value for the maximum range $\left(u=9.9 \times 10^{-5}\right)$ obtained by Morton and Chung (1959) for this disease.

Recently we tried to locate patients with muscular dystrophy in three villages near Gwalior, Ujiain (Agar families included) and 4 affected propositi were recorded; mutation was indicated only in two of these families. In all there were 41 family members ( 24 males) and 6 patients with muscular dystrophy. In another survey 8 rural families were interviewed at Jabalpur. In one family the disease appeared only in the last generation. This family provided data on 21 people ( 10 males) and included 5 patients with muscular dystrophy.

The entire survey included 7 families but mutation was indicated only in three. These 3 families included 62 normal members (including 34 males) and 11 patients with muscular dystrophy.

The mutation rates so far reported for this disease from different populations vary from $4 \times 10^{-5}$ 
TABLE

\begin{tabular}{|c|c|c|c|c|c|c|c|c|c|}
\hline \multirow{2}{*}{ Pedigree No. } & \multirow{2}{*}{ Age } & \multicolumn{8}{|c|}{ Relatives (age) } \\
\hline & & Father & Mother & $\begin{array}{c}\text { Maternal } \\
\text { Uncles }\end{array}$ & Brother(s) & Sister(s) & Husband & Son $(s)$ & Daughter(s) \\
\hline $\mathrm{I} .2$ & 98 & & & & & & $79 *$ & $81,76,74$, and 72 & \\
\hline II.5 & 78 & & & & 77,72 , and 68 & & 81 & & \\
\hline III.2 & 56 & & & & 54 & & 59 & $39,37,32$, and 30 & \\
\hline IV.5 & 34 & & & & 30 and 28 & 32 and $27 t$ & 39 & $10^{*}, 7$, and 5 & \\
\hline IV.9 & 27 & 53 & 50 & 53 and 51 & & & 30 & 8 and 6 & 3 \\
\hline
\end{tabular}

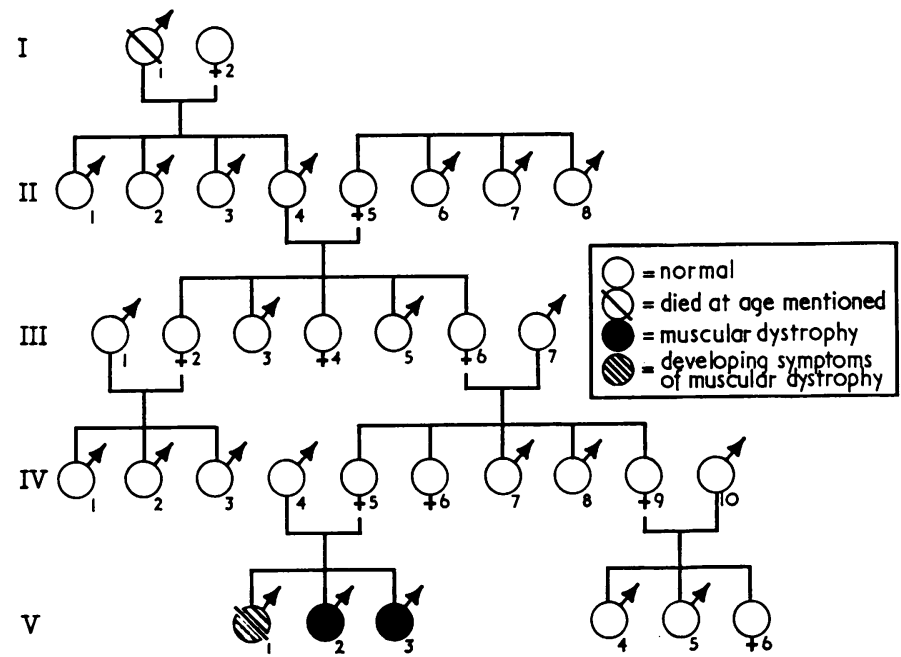

FIG. Family pedigree (cases IV.9 and IV.10 were distantly related).

to $9 \cdot 7 \times 10^{-5}$. The mutation rate calculated by the indirect method based on birth statistics of this sample $\left(9.7 \times 10^{-5}\right)$ is in agreement with the maximum range reported by Morton and Chung (1959). However according to Crow (1961) the minimum number of loci for muscular dystrophy is 2 , and a lower mutation rate $\left(3.4 \times 10^{-5}\right.$ per locus) was suggested.

This merely indicates the wide variance in the results obtained when estimating mutation rates of the same disease and is probably due to imperfections in either method of calculation.

\section{Summary}

In a survey, 2 patients with muscular dystrophy were found to have been born in a normal family. The mutation rate calculated by the indirect method based on available birth statistics was found to be $9.7 \times 10^{-5}$, which is in close agreement with results obtained by other workers.

An extension of the survey recorded 11 patients with muscular dystrophy born in 3 families. As statistics relating to births and deaths were not available (as is common in most Indian samples) it was impractical to calculate the population incidence and the mutation rate is not estimated from these samples.

We are grateful to Dr B. S. Bhatnagar and Mrs Dabar, the Medical Officers in Agar, various Sanitary Inspectors, the family members, and the patients for their hearty cooperation.

Thanks are also due to Professors L. S. Penrose and A. S. Sorsby (London), the late Professor S. S. Sarkar (Calcutta), and the National Library of Medicine (USA) for encouragement and help with the references.

row, J. F. (1961). Mutation in man. Progress in Medical Genetics, 1, 1-26.

Haldane, J. B. S. (1965). The implications of genetics for human society. Proceedings of the XIIth International Genetics Congress.

Morton, N. E. and Chung, C. S. (1959). Formal genetics of muscular dystrophy. American fournal of Human Genetics, 11, 360-379.

Penrose, L. S. (1961). Mutation. In Recent Advances in Human Genetics, edited by L. S. Penrose and A. L. Brown, pp. 1-18, Churchill, London.

Snyder, L. H. (1941). Medical Genetics. Duke University Press Durham NC.

Sutton, H. E. (1965). An Introduction to Human Genetics. Holt Rinehart and Winston, New York. 